PROCEEDINGS OF THE

AMERICAN MATHEMATICAL SOCIETY

Volume 130, Number 8, Pages 2275-2285

S 0002-9939(02)06627-3

Article electronically published on March 13, 2002

\title{
PERTURBATIONS OF EXISTENCE FAMILIES FOR ABSTRACT CAUCHY PROBLEMS
}

\author{
TI-JUN XIAO AND JIN LIANG
}

(Communicated by Jonathan M. Borwein)

\begin{abstract}
In this paper, we establish Desch-Schappacher type multiplicative and additive perturbation theorems for existence families for arbitrary order abstract Cauchy problems in a Banach space: $u^{(n)}(t)=A u(t)(t \geq 0)$; $u^{(j)}(0)=x_{j}(0 \leq j \leq n-1)$. As a consequence, we obtain such perturbation results for regularized semigroups and regularized cosine operator functions. An example is also given to illustrate possible applications.
\end{abstract}

\section{INTRODUCTION}

Notations. $N, R, R^{+}$, and $\mathbf{C}$ denote the positive integers, the real numbers, the nonnegative real numbers, and the complex numbers respectively. For Banach spaces $X$ and $Y, \mathbf{L}(X, Y)$ denotes the space of all bounded linear operators from $X$ to $Y$, and $\mathbf{L}(X):=\mathbf{L}(X, X)$. For a closed linear operator $A$, we write $\mathcal{D}(A), \mathcal{R}(A)$, and $\rho(A)$ for the domain, the range, and the resolvent set of $A$ respectively, and $[\mathcal{D}(A)]$ for the Banach space $\mathcal{D}(A)$ with the graph norm $\|x\|_{[\mathcal{D}(A)]}:=\|x\|+\|A x\|$. For $k \in N \cup\{0\}, C^{k}\left(R^{+}, X\right)$ is the set of all $k$-times continuously differentiable $X$-valued functions in $R^{+}$, and $C\left(R^{+}, X\right):=C^{0}\left(R^{+}, X\right) . M$ and $\omega$ will be positive constants. For a function $F:(\omega, \infty) \rightarrow \mathbf{L}(X), F \in L T-\mathbf{L}(X)$ means that there exists a strongly continuous and exponentially bounded function $H(\cdot): R^{+} \rightarrow \mathbf{L}(X)$ such that $F(\lambda) x=\int_{0}^{\infty} e^{-\lambda t} H(t) x d t(\lambda>\omega, x \in X)$.

Let $X$ be a Banach space, and let $C \in \mathbf{L}(X)$ be injective. Based on Lions [15] and his own paper 2, Da Prato 3] introduced the $C$-regularized semigroup on $X$ in 1966. Since Davies and Pang (4]) rediscovered it in 1987, this semigroup has been extensively investigated (cf., e.g., 6, 7, 11, 12, 17, 21, 22]), because it can be used to deal with many ill-posed (in the classical sense) abstract Cauchy problems for which the strongly continuous semigroup is not applicable. In 1991, a new type of operator family, called the existence family, for controlling the first order abstract Cauchy problem, which is more general than the $C$-regularized semigroup, was introduced and discussed by deLaubenfels [5] (see also [6]). It proves to be more flexible in applications, because the existence family does not require commutativity

Received by the editors November 8, 2000.

2000 Mathematics Subject Classification. Primary 47D06; Secondary 34G10.

Key words and phrases. Existence family, abstract Cauchy problem, regularized semigroups, regularized cosine operator functions, perturbation.

This work was supported partly by the NSF of China, the Key-Project-Foundation of the Chinese Academy of Sciences, and the Ministry of Education of China.

(C)2002 American Mathematical Society 
among itself, its generator, and the regularizing operator (cf. [5, 6]). The present paper is concerned with this type of operator family. Our focus is to study the Desch-Schappacher type multiplicative and additive perturbations for the existence family. To make the results more meaningful, we will carry out our discussion in a more general setting. Explicitly, we will study the multiplicative and additive perturbations for existence families for arbitrary order abstract Cauchy problems:

$$
u^{(n)}(t)=A u(t)(t \geq 0), \quad u^{(j)}(0)=x_{j}(0 \leq j \leq n-1),
$$

where $n \in N$, and $A$ is a closed linear operator in $X$.

Definition 1.1. The strongly continuous family of operators $\{S(t)\}_{t>0} \subset \mathbf{L}(X)$ is called a $C$-existence family for (1.1), if for all $x \in X$ and $t \geq 0$ we have $S(\cdot) x \in$ $C^{n-1}\left(R^{+}, X\right), A \int_{0}^{t} S(s) x d s \in C\left(R^{+}, X\right)$, and

$$
S(t) x=\frac{t^{n-1}}{(n-1) !} C x+A \int_{0}^{t} \frac{(t-s)^{n-1}}{(n-1) !} S(s) x d s .
$$

We also say that (1.1) has a $C$-existence family $\{S(t)\}_{t \geq 0}$.

It is known from [6, Chapter III] that the $C$-existence family reduces to a $C$ regularized semigroup when $n=1$ and $S(t) A \subset A S(t)(t \geq 0)$. Moreover, letting $n=2$ and $S(t) A \subset A S(t)$ in Definition 1.1 gives the $C$-regularized cosine operator function $\left\{S^{\prime}(t)\right\}_{t \geq 0}$.

The Desch-Schappacher perturbations were firstly studied in [8] for classical strongly continuous semigroups in 1989. In recent years, this type of perturbations has drawn many researchers' attention, and the related theory has been gaining much development (cf., e.g., Engel and Nagel [10, Section III.3], [1, 7, 9, 13, 18, 19 20, and references therein). In [9], Diekmann, Gyllenberg and Thieme showed a new viewpoint of perturbations of Desch-Schappacher type, by solving Stieltjes' renewal equations with the basic assumption on the behaviour of the semivariation of the step response function (see also [19]). In [13, Jung investigated how certain properties, like analyticity or norm continuity, of the original semigroup are inherited by the perturbed semigroup. In [7, Section V] by deLaubenfels and Yao, nonlinear additive perturbations of this type for $C$-regularized semigroups were discussed, and a local existence and uniqueness theorem on the classical solutions of the Cauchy problem for the associated perturbed equation was given. Moreover, in 20, 1, 18, one can see results about such perturbations for classical strongly continuous cosine operator functions, and for solution families or $n$-times integrated solution families of linear Volterra equations.

In this paper, we will present Desch-Schappacher type multiplicative and additive perturbation theorems for the general existence family given by Definition 1.1, and show the uniqueness of solutions for the corresponding perturbed (1.1) (Theorems 2.1 and 2.2). As a consequence, we obtain Desch-Schappacher type perturbation theorems for regularized semigroups and regularized cosine operator functions (Corollaries 2.3, 2.4 and 2.6), which recover the corresponding results in [5, 6, 8, 19, 20] (see Remarks 2.5 and 2.7). With a new observation of the ranges of perturbation operators, we exhibit in Theorem 2.8 two classes of perturbation operators satisfying the conditions of Theorem 2.1 or Theorem 2.2. Finally, an example (Example 2.9) is given to illustrate possible applications. This example also reflects the features of Theorem 2.8 (see Remark 2.10). 
The following characterization and properties of exponentially bounded existence families will be used in the sequel. By a solution of (1.1), we mean a function $u(\cdot) \in C^{n}\left(R^{+}, X\right) \cap C\left(R^{+},[\mathcal{D}(A)]\right)$ satisfying (1.1).

Proposition $1.2([23])$. Let $\lambda^{n}-A$ be injective for $\lambda>\omega$. Then (1.1) has a $C$ existence family $\{S(t)\}_{t \geq 0}$ on $X$ with $\left\|S^{(n-1)}(t)\right\| \leq M e^{\omega t}(t \geq 0)$ if and only if $\mathcal{R}(C) \subset \mathcal{R}\left(\lambda^{n}-A\right)$ for $\lambda>\omega$, and the function $\lambda \mapsto \lambda^{n-1}\left(\lambda^{n}-A\right)^{-1} C \in$ $L T-\mathbf{L}(X)$; in this case, for $x_{j} \in \mathcal{D}(A)$ with $A x_{j} \in \mathcal{R}(C)(0 \leq j \leq n-1),(1.1)$ admits a solution $u(\cdot)$ satisfying

$$
\left\|u^{(n)}(t)\right\|,\|u(t)\|_{[\mathcal{D}(A)]} \leq M e^{\omega t} \sum_{i=0}^{n-1}\left(\left\|u_{i}\right\|+\left\|C^{-1} A u_{i}\right\|\right), \quad t \geq 0,
$$

and

$$
\lambda^{n-1}\left(\lambda^{n}-A\right)^{-1} C x=\int_{0}^{\infty} e^{-\lambda t} S^{(n-1)}(t) x d t, \quad x \in X, \lambda>\omega .
$$

\section{Results AND PROOFS}

Theorem 2.1. Let $A$ and $\{S(t)\}_{t \geq 0}$ be as in Proposition 1.2 , and let $\alpha, \beta \in \mathbf{C}$. Suppose $B \in \mathbf{L}(X)$ and $\mathcal{R}(B) \subset \mathcal{R}(C)$. If for every $f \in C\left(R^{+}, X\right)$ and $t \geq 0$,

$$
\left\|A \int_{0}^{t} S(t-s) C^{-1} B f(s) d s\right\| \leq M \int_{0}^{t} e^{\omega(t-s)}\|f(s)\| d s,
$$

then

(i) the Cauchy problem

$$
u^{(n)}(t)=(A(I+\alpha B)+\beta B) u(t)(t \geq 0), \quad u^{(j)}(0)=x_{j}(0 \leq j \leq n-1)
$$

has a $C$-existence family $\{U(t)\}_{t \geq 0}$ on $X$ and $\left\|U^{(n-1)}(\cdot)\right\|$ is exponentially bounded; and

(ii) all solutions of (2.2) are unique, provided $C A \subset A C$.

Proof. Fixing $f \in C\left(R^{+}, X\right)$, by (2.1) and (1.2) we obtain that for $0 \leq t_{2} \leq t_{1}<\infty$,

$$
\begin{aligned}
& \left\|A \int_{0}^{t_{1}} S\left(t_{1}-s\right) C^{-1} B f(s) d s-A \int_{0}^{t_{2}} S\left(t_{2}-s\right) C^{-1} B f(s) d s\right\| \\
\leq & \left\|A \int_{0}^{t_{1}} S\left(t_{1}-s\right) C^{-1} B\left[f(s)-f\left(s-t_{1}+t_{2}\right)\right] d s\right\| \\
& +\left\|A \int_{0}^{t_{1}-t_{2}} S\left(t_{1}-s\right) C^{-1} B f\left(s-t_{1}+t_{2}\right) d s\right\| \\
\leq & M e^{\omega t_{1}} \max _{0 \leq s \leq t_{1}}\left\|f(s)-f\left(s-t_{1}+t_{2}\right)\right\|+\left\|A \int_{t_{2}}^{t_{1}} S(s) C^{-1} B f(0) d s\right\| \\
\leq & M e^{\omega t_{1}} \max _{0 \leq s \leq t_{1}}\left\|f(s)-f\left(s-t_{1}+t_{2}\right)\right\|+\left\|\left[S^{(n-1)}\left(t_{1}\right)-S^{(n-1)}\left(t_{2}\right)\right] C^{-1} B f(0)\right\|,
\end{aligned}
$$

where $f(-s):=f(0)$ for $s>0$; this implies that the function

$$
t \mapsto A \int_{0}^{t} S(t-s) C^{-1} B f(s) d s \in C\left(R^{+}, X\right)
$$


$\left(f \in C\left(R^{+}, X\right)\right)$. We set $W_{0}(t)=S^{(n-1)}(t)(t \geq 0)$ and define $W_{n}(t)$ inductively by

$$
W_{n}(t) x=(\beta+\alpha A) \int_{0}^{t} S(t-s) C^{-1} B W_{n-1}(s) x d s, \quad x \in X, t \geq 0, n \in N .
$$

Clearly, $\left\{W_{n}(t)\right\}_{t \geq 0}$ is a strongly continuous family of bounded linear operators on $X$, for each $n \in N$. We know by hypothesis that $S(\cdot)$ and $W_{0}(\cdot)$ are exponentially bounded. So using (2.1) we get by induction that $\left\|W_{n}(t)\right\| \leq M_{1}^{n+1} e^{\omega_{1} t} \frac{t^{n}}{n !}(t \geq 0$, $n \in N \cup\{0\}$ ), for certain constants $M_{1}>M, \omega_{1}>\omega$. Define $W(t):=\sum_{n=0}^{\infty} W_{n}(t)$ $(t \geq 0)$. We see by the above arguments that the series converges in the uniform operator topology, uniformly for bounded intervals of $R^{+},\|W(t)\| \leq M_{1} e^{\left(\omega_{1}+M_{1}\right) t}$ $(t \geq 0)$, and hence $\{W(t)\}_{t \geq 0} \subset \mathbf{L}(X)$ is a strongly continuous family. Thus, by (2.3) we have

$$
W(t) x=S^{(n-1)}(t) x+(\beta+\alpha A) \int_{0}^{t} S(t-s) C^{-1} B W(s) x d s, \quad x \in X, t \geq 0 .
$$

Taking Laplace transforms, we obtain by (1.3) that, for $\lambda$ large enough and $x \in X$,

$$
\begin{aligned}
\int_{0}^{\infty} & e^{-\lambda t} W(t) x d t \\
& =\lambda^{n-1}\left(\lambda^{n}-A\right)^{-1} C x+(\beta+\alpha A)\left(\lambda^{n}-A\right)^{-1} B \int_{0}^{\infty} e^{-\lambda t} W(t) x d t .
\end{aligned}
$$

Therefore, for such $\lambda$,

$$
\left(\lambda^{n}-A(I+\alpha B)-\beta B\right) \int_{0}^{\infty} e^{-\lambda t} W(t) x d t=\lambda^{n-1} C x, \quad x \in X,
$$

by the equalities

$$
\begin{aligned}
& \left(\lambda^{n}-A\right)\left[I-(\beta+\alpha A)\left(\lambda^{n}-A\right)^{-1} B\right] \\
& \quad=\left(\lambda^{n}-A\right)\left[I+\alpha B-\alpha \lambda^{n}\left(\lambda^{n}-A\right)^{-1} B-\beta\left(\lambda^{n}-A\right)^{-1} B\right] \\
& \quad=\lambda^{n}-A(I+\alpha B)-\beta B .
\end{aligned}
$$

Finally, we show that $I-(\beta+\alpha A)\left(\lambda^{n}-A\right)^{-1} B$ is invertible for large $\lambda$. In order to do this, we observe by (2.1) and (1.3) that for each $x \in X, t \geq 0$,

$$
\begin{gathered}
\left\|(\beta+\alpha A) \int_{0}^{t} S(t-s) C^{-1} B x d s\right\| \leq M_{2} \int_{0}^{t} e^{\omega_{2}(t-s)}\|x\| d s \leq \frac{M_{2}}{\omega_{2}}\left(e^{\omega_{2} t}-1\right)\|x\|, \\
(\beta+\alpha A)\left(\lambda^{n}-A\right)^{-1} B x \\
=\lambda \int_{0}^{\infty} e^{-\lambda t}\left[(\beta+\alpha A) \int_{0}^{t} S(t-s) C^{-1} B x d s\right] d t, \quad \lambda>\omega_{2},
\end{gathered}
$$

where $M_{2}$ and $\omega_{2}$ are positive constants. So for $\lambda>\omega_{2}$ and $x \in X$,

$$
\left\|(\beta+\alpha A)\left(\lambda^{n}-A\right)^{-1} B x\right\| \leq \frac{M_{2} \lambda}{\omega_{2}} \int_{0}^{\infty} e^{-\lambda t}\left(e^{\omega_{2} t}-1\right)\|x\| d t=\frac{M_{2}\|x\|}{\lambda\left(\lambda-\omega_{2}\right)} .
$$

Thus for $\lambda>2 M_{2}+\omega_{2}+1$ we have $\left\|(\beta+\alpha A)\left(\lambda^{n}-A\right)^{-1} B\right\|<\frac{1}{2}$, so that

$$
I-(\beta+\alpha A)\left(\lambda^{n}-A\right)^{-1} B
$$


is invertible. This together with (2.5) yields that for $\lambda>2 M_{2}+\omega_{2}+\omega+1$ the operator $\lambda^{n}-A(I+\alpha B)-\beta B$ is injective, since $\lambda^{n}-A$ is injective for $\lambda>\omega$. In conclusion, we obtain from (2.4) that for $\lambda$ sufficiently large,

$$
\lambda^{n-1}\left(\lambda^{n}-A(I+\alpha B)-\beta B\right)^{-1} C x=\int_{0}^{\infty} e^{-\lambda t} W(t) x d t \quad(x \in X) .
$$

Set, for $t \geq 0$ and $x \in X$,

$$
U(t) x=\left\{\begin{array}{lll}
W(t) x, & \text { if } & n=1, \\
\int_{0}^{t} \frac{(t-s)^{n-2}}{(n-2) !} W(s) x d s, & \text { if } & n \geq 2 .
\end{array}\right.
$$

Then an application of Proposition 1.2 gives assertion (i).

In order to verify assertion (ii), we let $v(\cdot)$ be a solution of (2.2) with initial data $x_{j}=0(0 \leq j \leq n-1)$. Evidently

$$
v(t)=(A(I+\alpha B)+\beta B) \int_{0}^{t} \frac{(t-\sigma)^{n-1}}{(n-1) !} v(\sigma) d \sigma, \quad t \geq 0 .
$$

The assumption $C A \subset A C$ implies

$$
S(t) C=C S(t)(t \geq 0), \quad S(t) A x=A S(t) x(x \in \mathcal{D}(A), t \geq 0),
$$

according to (1.3) and the uniqueness theorem for Laplace transforms. So (1.2) yields

$$
S^{(n)}(t) x=S(t) A x, \quad x \in \mathcal{D}(A), t \geq 0 .
$$

Thus, by (2.6) - (2.8) we obtain that for $t \geq s \geq 0$,

$$
\begin{aligned}
& \frac{d}{d s}\left[\sum_{i=0}^{n-1} S^{(i)}(t-s)(I+\alpha B) \int_{0}^{s} \frac{(s-\sigma)^{i}}{i !} v(\sigma) d \sigma\right] \\
= & S(t-s)(I+\alpha B) v(s)+\sum_{i=1}^{n-1} S^{(i)}(t-s)(I+\alpha B) \int_{0}^{s} \frac{(s-\sigma)^{i-1}}{(i-1) !} v(\sigma) d \sigma \\
& -\sum_{i=0}^{n-1} S^{(i+1)}(t-s)(I+\alpha B) \int_{0}^{s} \frac{(s-\sigma)^{i}}{(i-1) !} v(\sigma) d \sigma \\
= & S(t-s)(I+\alpha B) v(s)-S^{(n)}(t-s)(I+\alpha B) \int_{0}^{s} \frac{(s-\sigma)^{n-1}}{(n-1) !} v(\sigma) d \sigma \\
= & C S(t-s)\left[\alpha C^{-1} B v(s)+\beta C^{-1} B \int_{0}^{s} \frac{(s-\sigma)^{n-1}}{(n-1) !} v(\sigma) d \sigma\right] .
\end{aligned}
$$

Noting that

$$
S^{(n-1)}(0)=I, \quad S^{(i)}(0)=0(0 \leq i \leq n-2)
$$

from (1.2), we then infer that for $t \geq 0$,

$$
\begin{aligned}
& C(I+\alpha B) \int_{0}^{t} \frac{(t-\sigma)^{n-1}}{(n-1) !} v(\sigma) d \sigma \\
= & C \int_{0}^{t} S(t-s)\left[\alpha C^{-1} B v(s)+\beta C^{-1} B \int_{0}^{s} \frac{(s-\sigma)^{n-1}}{(n-1) !} v(\sigma) d \sigma\right] d s .
\end{aligned}
$$


Since $C$ is injective, it follows from (2.6) that for $t \geq 0$,

$$
\begin{gathered}
v(t)=\beta B \int_{0}^{t} \frac{(t-\sigma)^{n-1}}{(n-1) !} v(\sigma) d \sigma+\alpha A \int_{0}^{t} S(t-\sigma) C^{-1} B v(\sigma) d \sigma \\
+\beta A \int_{0}^{t} S(t-\sigma) C^{-1} B\left(\int_{0}^{\sigma} \frac{(\sigma-\tau)^{n-1}}{(n-1) !} v(\tau) d \tau\right) d \sigma .
\end{gathered}
$$

Fix $T>0$. Then by (2.1) there exists a constant $M_{0}>0$ such that for each $t \in[0, T], \max _{0 \leq s \leq t}\|v(s)\| \leq M_{0} \int_{0}^{t} \max _{0 \leq \tau \leq \sigma}\|v(\tau)\| d \sigma$. So the Gronwall-Bellman inequality shows that $v(t)=0$ for $t \in[0, T]$. Because $T$ was arbitrary, $v(t) \equiv 0$ for $t \geq 0$. This ends the proof.

Theorem 2.2. Let $A$ and $\{S(t)\}_{t \geq 0}$ be as in Proposition 1.2. Suppose $\mathbf{B}$ is a closed linear operator in $X$ such that $\mathcal{D}(\mathbf{B}) \supset \mathcal{D}(A)$ and $\mathcal{R}(\mathbf{B}) \subset \mathcal{R}(C)$. If for each $f \in C\left(R^{+},[\mathcal{D}(A)]\right)$ and $t \geq 0$ we have

$$
\left\|A \int_{0}^{t} S(t-s) C^{-1} \mathbf{B} f(s) d s\right\| \leq M \int_{0}^{t} e^{\omega(t-s)}\|f(s)\|_{[\mathcal{D}(A)]} d s,
$$

then

(i) the Cauchy problem

$$
u^{(n)}(t)=(A+\mathbf{B}) u(t)(t \geq 0), \quad u^{(j)}(0)=x_{j}(0 \leq j \leq n-1)
$$

has a C-existence family $\{V(t)\}_{t \geq 0}$ on $[\mathcal{D}(A)]$, and $\left\|V^{(n-1)}(\cdot)\right\|_{\mathbf{L}([\mathcal{D}(A)])}$ is exponentially bounded;

(ii) for any $x_{j} \in C(\mathcal{D}(A))(0 \leq j \leq n-1)$, the function $\sum_{j=0}^{n-1} V^{(n-1-j)}(\cdot) C^{-1} x_{j}$ is a solution of (2.11); and

(iii) all solutions of (2.11) are unique, provided $C A \subset A C$.

Proof. Define $Y(t)=\sum_{n=0}^{\infty} Y_{n}(t)(t \geq 0)$, where $Y_{0}(t)=S^{(n-1)}(t)$ and $Y_{n}(t) x=$ $\int_{0}^{t} S(t-s) C^{-1} \mathbf{B} Y_{n-1}(s) x d s(t \geq 0, x \in \mathcal{D}(A), n \in N)$. Then, arguing similarly as in the proof of Theorem 2.1, we obtain that $\{Y(t)\}_{t \geq 0}$ is an exponentially bounded, strongly continuous family of bounded linear operators on $[\mathcal{D}(A)]$, and for $\lambda$ large enough, $\left(\lambda^{n}-(A+\mathbf{B})\right) \int_{0}^{\infty} e^{-\lambda t} Y(t) x d t=\lambda^{n-1} C x(x \in \mathcal{D}(A))$, and

$$
\left\|\left(\lambda^{n}-A\right)^{-1} \mathbf{B}\right\|_{\mathbf{L}([\mathcal{D}(A)])}<\frac{1}{2},
$$

so that $\lambda^{n}-(A+\mathbf{B})$ is injective and

$$
\lambda^{n-1}\left(\lambda^{n}-(A+\mathbf{B})\right)^{-1} C x=\int_{0}^{\infty} e^{-\lambda t} Y(t) x d t, \quad x \in \mathcal{D}(A) .
$$

Therefore, (2.11) has a $C$-existence family $\{V(t)\}_{t \geq 0}$ on $[\mathcal{D}(A)]$, given by

$$
V(t) x:=\left\{\begin{array}{llc}
Y(t) x, & \text { if } & n=1, \\
\int_{0}^{t} \frac{(t-s)^{n-2}}{(n-2) !} Y(s) x d s, & \text { if } & n \geq 2,
\end{array}\right.
$$

in view of Proposition 1.2. This completes the proof of part (i).

Next, from the above conclusion we have

$$
\begin{aligned}
V(t) x-\frac{t^{n-1}}{(n-1) !} C x & =(A+\mathbf{B}) \int_{0}^{t} \frac{(t-s)^{n-1}}{(n-1) !} V(s) x d s \\
& =\int_{0}^{t} \frac{(t-s)^{n-1}}{(n-1) !}(A+\mathbf{B}) V(s) x d s, \quad x \in \mathcal{D}(A), t \geq 0 .
\end{aligned}
$$


This leads to part (ii) immediately.

To prove part (iii) we let $w(\cdot)$ be a solution of (2.11) with $x_{j}=0$ for all $0 \leq$ $j \leq n-1$. By (2.7) and (2.8) we deduce that $\frac{d}{d s}\left[\sum_{i=0}^{n-1} S^{(i)}(t-s) w^{(n-1-i)}(s)\right]=$ $C S(t-s) C^{-1} \mathbf{B} w(s)(t \geq s \geq 0)$, so that $w(t)=\int_{0}^{t} S(t-s) C^{-1} \mathbf{B} w(s) d s(t \geq 0)$ by (2.9). Thus from (2.10) we get $\left\|e^{-\omega t} w(t)\right\|_{[\mathcal{D}(A)]} \leq M^{\prime} \int_{0}^{t}\left\|e^{-\omega t} w(t)\right\|_{[\mathcal{D}(A)]} d s$ $(t \geq 0)$, for some constant $M^{\prime}>0$. It follows that $w(t) \equiv 0$ for $t \geq 0$ by using the Gronwall-Bellman inequality. The proof is complete.

In what follows, we give multiplicative and additive perturbation theorems with regard to exponentially bounded regularized semigroups and regularized cosine operator functions, as consequences of Theorems 2.1 and 2.2. Let $A$ and $\{S(t)\}_{t \geq 0}$ be as in Proposition 1.2. If $n=1$ (resp. $n=2$ ) and $C A \subset A C$, then $S(\cdot)$ (resp. $C(\cdot):=S^{\prime}(\cdot)$ ) is an exponentially bounded $C$-regularized semigroup (resp. cosine operator function), with $C^{-1} A C$ as its generator. In this case, $A$ is called a subgenerator of $S(\cdot)$ (resp. $C(\cdot)$ ); in other words, $A$ subgenerates $S(\cdot)$ (resp. $C(\cdot)$ ). For more information on regularized semigroups and regularized cosine operator functions, we refer to, e.g., [6, 16, 22] and references therein.

Corollary 2.3. Assume that A subgenerates an exponentially bounded $C$-regularized semigroup $\{S(t)\}_{t \geq 0}$ (resp. cosine operator function $\{C(t)\}_{t \geq 0}$ ) on $X$. Let $\alpha, \beta \in \mathbf{C}$, and $B \in \mathbf{L}(X)$ with $\mathcal{R}(B) \subset \mathcal{R}(C)$, and let $C_{1} \in \mathbf{L}(X)$ be injective such that $\mathcal{R}\left(C_{1}\right) \subset \mathcal{R}(C)$ and $C_{1}[A(I+\alpha B)+\beta B] \subset[A(I+\alpha B)+\beta B] C_{1}$. If (2.1) holds (in the case of the cosine operator function, $\left.S(t) x:=\int_{0}^{t} C(s) x d s\right)$, then $A(I+\alpha B)+\beta B$ subgenerates an exponentially bounded $C_{1}$-regularized semigroup (resp. cosine operator function) on $X$.

Proof. Apply Theorem 2.1. $U(t) C^{-1} C_{1}$ (resp. $U^{\prime}(t) C^{-1} C_{1}$ ) is the $C_{1}$-regularized semigroup (resp. cosine operator function), as claimed.

Corollary 2.4. Assume that A subgenerates an exponentially bounded $C$-regularized semigroup $\{S(t)\}_{t \geq 0}$ (resp. cosine operator function $\{C(t)\}_{t \geq 0}$ ) on $X$. Let $\mathbf{B}$ be a closed linear operator in $X$ such that $\mathcal{D}(\mathbf{B}) \supset \mathcal{D}(A)$ and $\mathcal{R}(\mathbf{B}) \subset \mathcal{R}(C)$. Let $C_{1} \in \mathbf{L}(X)$ be injective such that $\mathcal{R}\left(C_{1}\right) \subset \mathcal{R}(C), C^{-1} C_{1}: \mathcal{D}(A) \rightarrow \mathcal{D}(A)$, and $C_{1}(A+\mathbf{B}) \subset(A+\mathbf{B}) C_{1}$. If (2.10) holds (in the case of the cosine operator function, $\left.S(t) x:=\int_{0}^{t} C(s) x d s\right)$, then $A+\mathbf{B}$ subgenerates an exponentially bounded $C_{1}$-regularized semigroup (resp. cosine operator function) on $X$, provided that $\rho(A)$ contains a sequence of real numbers, tending to $+\infty$.

Proof. From (2.12) we see that there exists a $\mu_{0} \in \rho(A)$ such that

$$
\left\|\left(\mu_{0}-A\right)^{-1} \mathbf{B}\right\|_{\mathbf{L}([\mathcal{D}(A))]}<\frac{1}{2}
$$

and therefore $\mu_{0}-(A+\mathbf{B})=\left(\mu_{0}-A\right)\left(I-\left(\mu_{0}-A\right)^{-1} \mathbf{B}\right)$ is invertible on $X$. Letting $Y(\cdot)$ be as in (2.13) with $n=1$ (resp. $n=2$ ), we put

$$
\tilde{Y}(t):=\left[\mu_{0}-(A+\mathbf{B})\right] Y(t) C^{-1} C_{1}\left[\mu_{0}-(A+\mathbf{B})\right]^{-1} \quad(t \geq 0) .
$$


Then $\{\tilde{Y}(t)\}_{t \geq 0}$ is a strongly continuous family of operators in $\mathbf{L}(X)$, and for $\lambda$ large enough,

$$
\begin{aligned}
\int_{0}^{\infty} e^{-\lambda t} \tilde{Y}(t) x d t & =\lambda^{n-1}\left[\mu_{0}-(A+\mathbf{B})\right]\left[\lambda^{n}-(A+\mathbf{B})\right]^{-1} C_{1}\left[\mu_{0}-(A+\mathbf{B})\right]^{-1} \\
& =\lambda^{n-1}\left[\lambda^{n}-(A+\mathbf{B})\right]^{-1} C_{1} x, \quad t \geq 0, x \in X,
\end{aligned}
$$

with $n=1$ (resp. $n=2$ ).

Remark 2.5. For the case when $C=C_{1}=I, \alpha=1, \beta=0$, and $A$ generates a strongly continuous semigroup $\{S(t)\}_{t \geq 0}$ (resp. strongly continuous cosine operator function $\left.\{C(t)\}_{t \geq 0}\right)$ on $X$, Corollaries 2.3 and 2.4 can be found in $8,19,20$. In this case, $\{S(t)\}_{t \geq 0}$ (resp. $\left.\{C(t)\}_{t \geq 0}\right)$ is exponentially bounded and $\rho(A)$ contains a right half plane, automatically.

It is plain to see that (2.1) holds for

$$
B \in \mathbf{L}(X) \quad \text { with } \mathcal{R}(B) \subset \mathcal{D}\left(A C^{-1}\right),
$$

and that (2.10) holds for any closed linear operator $\mathbf{B}$ in $X$ with $\mathcal{D}(\mathbf{B}) \supset \mathcal{D}(A)$ and $\mathcal{R}(\mathbf{B}) \subset \mathcal{D}\left(A C^{-1}\right)$. Specifically, we have the following result.

Corollary 2.6. Suppose that $A$ subgenerates an exponentially bounded $C$-regularized semigroup on $X$, and that $B_{1} \in \mathbf{L}(X)$ and $\mathcal{R}\left(B_{1}\right) \subset \mathcal{R}(C)$. Then:

(i) The Cauchy problem $(*): u^{\prime}(t)=\left(A+B_{1}\right) u(t)(t \geq 0), u(0)=x$ has an exponentially bounded $C$-existence family on $X$.

(ii) All solutions of $(*)$ are unique, provided $C A \subset A C$.

(iii) $A+B_{1}$ subgenerates an exponentially bounded $C_{1}$-regularized semigroup on $X$, whenever $C_{1} \in \mathbf{L}(X)$ is injective, $\mathcal{R}\left(C_{1}\right) \subset \mathcal{R}(C)$ and $C_{1}\left(A+B_{1}\right) \subset$ $\left(A+B_{1}\right) C_{1}$.

Proof. Take $\alpha=0$ and $\beta=1$ in Theorem 2.1 and Corollary 2.3.

Remark 2.7. Conclusion (i) of Corollary 2.6 appeared in [5, 6]. Generally speaking, a $C$-existence family for a first order Cauchy problem ensures uniqueness of the exponentially bounded solutions, but not all solutions (see [6, Proposition 2.9]). This indicates the significance of assertion (ii). Conclusion (iii) is due to [21].

Let $A_{0}$ be a linear operator in $X$ satisfying

$$
(\omega, \infty) \subset \rho\left(A_{0}\right), \quad \sup _{\lambda>\omega}\left\|\lambda\left(\lambda-A_{0}\right)^{-1}\right\|<\infty .
$$

We set $F_{A_{0}}:=\left\{x \in X ; \varlimsup_{\lambda \rightarrow+\infty}\left\|\lambda A_{0}\left(\lambda-A_{0}\right)^{-1} x\right\|<\infty\right\}$. It is easy to verify that $F_{A_{0}}$, endowed with the norm $\|x\|_{F_{A_{0}}}:=\|x\|+\varlimsup_{\lim _{\lambda \rightarrow+\infty}}\left\|\lambda A_{0}\left(\lambda-A_{0}\right)^{-1} x\right\|$, is a Banach space. When $A_{0}$ is the generator of a strongly continuous semigroup $\{T(t)\}_{t \geq 0}, F_{A_{0}}$ coincides with the Favard class of $T(t)$, cf. [10, p.130, Proposition $5.12]$.

Theorem 2.8. Let $A$ and $\{S(t)\}_{t \geq 0}$ be as in Proposition 1.2, and let $C_{0} \in \mathbf{L}(X)$ with $C C_{0}=C_{0} C$ and $C_{0} A \subset A C_{0}$. Suppose $A_{0}$ is a densely defined linear operator in $X$ satisfying (2.15), such that $\mathcal{D}\left(A_{0}\right) \subset \mathcal{D}\left(A C_{0}\right), C A_{0} \subset A_{0} C$, and $\left(\lambda-A_{0}\right)^{-1} A \subset A\left(\lambda-A_{0}\right)^{-1}$ for $\lambda>\omega$. Then:

(i) (2.1) is valid for $B=C C_{0} B_{0}$, if $B_{0} \in \mathbf{L}(X)$ and $\mathcal{R}\left(B_{0}\right) \subset F_{A_{0}}$.

(ii) (2.10) is valid for $\mathbf{B}=C C_{0} \mathbf{B}_{0}$, if $\mathbf{B}_{0}$ is a closed linear operator in $X$, $\mathcal{D}\left(\mathbf{B}_{0}\right) \supset \mathcal{D}(A)$, and $\mathcal{R}\left(\mathbf{B}_{0}\right) \subset F_{A_{0}}$. 
Proof. Using the density of $\mathcal{D}\left(A_{0}\right)$ and (2.15), we have

$$
\lim _{\lambda \rightarrow+\infty} \lambda\left(\lambda-A_{0}\right)^{-1} x=x, \quad x \in X .
$$

Moreover, by hypothesis, $A_{0}\left(\lambda-A_{0}\right)^{-1}\left(\mu^{n}-A\right)^{-1} C=\left(\mu^{n}-A\right)^{-1} C A_{0}\left(\lambda-A_{0}\right)^{-1}$ $(\lambda, \mu>\omega)$. In combination with (1.3), this shows that for $\lambda>\omega, t \geq 0$,

$$
A_{0}\left(\lambda-A_{0}\right)^{-1} S(t)=S(t) A_{0}\left(\lambda-A_{0}\right)^{-1},
$$

by the uniqueness theorem for Laplace transforms. Likewise,

$$
C_{0} S(t)=S(t) C_{0}, \quad t \geq 0 .
$$

Let $B_{0}$ and $B$ be as in (i). For each $f \in C\left(R^{+}, X\right)$ and $t>0$, we take a sequence $\left\{f_{m}\right\}_{m \in N} \subset C^{1}([0, t], X)$ such that

$$
\max _{s \in[0, t]}\left\|f_{m}(s)-f(s)\right\| \rightarrow 0, \quad \text { as } m \rightarrow \infty .
$$

From [22], we know that

$$
\int_{0}^{t} S(t-s) C_{0} B_{0} f_{m}(s) d s \in \mathcal{D}(A), \quad m \in N .
$$

Therefore, noting that $\mathcal{D}\left(A C_{0}\right) \supset \mathcal{D}\left(A_{0}\right)$ and using (2.16) - (2.18), we obtain

$$
\begin{aligned}
& \left\|A \int_{0}^{t} S(t-s) C^{-1} B f_{m}(s) d s\right\|=\left\|A C_{0} \int_{0}^{t} S(t-s) B_{0} f_{m}(s) d s\right\| \\
\leq & \left\|A C_{0}\left(\omega+1-A_{0}\right)^{-1}\right\| \\
& \quad \times \lim _{\lambda \rightarrow+\infty}\left\|\int_{0}^{t} S(t-s)\left[\lambda\left(\omega+1-A_{0}\right)\left(\lambda-A_{0}\right)^{-1}\right] B_{0} f_{m}(s) d s\right\| \\
\leq & \widetilde{M} \int_{0}^{t} e^{\omega(t-s)}\left\|B_{0} f_{m}(s)\right\|_{F_{A_{0}}} d s \\
\leq & \widetilde{M}\left\|B_{0}\right\|_{\mathbf{L}\left(X, F_{A_{0}}\right)} \int_{0}^{t} e^{\omega(t-s)}\left\|f_{m}(s)\right\| d s, \quad m \in N,
\end{aligned}
$$

where $\widetilde{M}$ is a constant independent of $m$ and $t$. This proves part (i), by (2.19) and the closedness of $A$. The same type of argument gives part (ii).

Example 2.9. Let $X=U C_{b}(R)$ be the space of uniformly continuous and bounded functions, and let

$$
\begin{aligned}
& A=i \frac{d^{2}}{d \xi^{2}} \quad \text { with } \mathcal{D}(A)=\left\{f \in C^{2}(R) ; f \text { is bounded and } f^{\prime \prime} \in X\right\} \\
& A_{0}=\frac{d^{2}}{d \xi^{2}} \quad \text { with } \mathcal{D}\left(A_{0}\right)=\mathcal{D}(A) .
\end{aligned}
$$

It is known that the operator $D:=\frac{d^{2}}{d \xi^{2}}$ with domain $W^{2,1}(R)$ generates a strongly continuous semigroup on $L^{1}(R)$, and $A_{0}$ is the generator of its sun dual semigroup on $X$. Thus $F_{A_{0}}$ coincides with the domain of the adjoint operator of $D$ (cf. [10, p.135, Proposition 5.19]). Hence it is not hard to see that $F_{A_{0}}=$ $\left\{f \in C^{1}(R) ; f\right.$ is bounded and $f^{\prime}$ is Lipschitz continuous $\}$. From [14, we see that $A$ generates an exponentially bounded once integrated semigroup, and so generates an exponentially bounded $C$-regularized semigroup (cf. [6, Theorem 18.3]) for $C:=\left(1-A_{0}\right)^{-1}$. Moreover, define $B_{0}$ by $\left(B_{0} f\right)(\xi)=i g(\xi) \int_{a}^{b} f(\sigma) d \sigma(f \in X)$, 
where $g(\xi) \in F_{A_{0}}$ and $a, b \in R$. Then $B_{0} \in \mathbf{L}(X)$ and $\mathcal{R}\left(B_{0}\right) \subset F_{A_{0}}$. Taking $\alpha=1, \beta=-i, C_{0}=I, B=C C_{0} B_{0}$, we have $A(\alpha I+B)+\beta B=A-i B_{0}$. Applying Theorem 2.1, Theorem 2.8 and Proposition 1.2, we conclude that for each $\phi \in U C_{b}(R) \cap C^{4}(R)$ with $\phi^{(4)} \in U C_{b}(R)$, the Cauchy problem

$$
\left\{\begin{array}{l}
\frac{\partial u(t, \xi)}{\partial t}=i \frac{\partial^{2} u(t, \xi)}{\partial \xi^{2}}+g(\xi) \int_{a}^{b} u(t, \sigma) d \sigma, \quad t \geq 0, \xi \in R \\
u(0, \xi)=\phi(\xi), \quad \xi \in R
\end{array}\right.
$$

has a unique solution in $C^{1}\left(R, U C_{b}(R)\right)$.

Remark 2.10. Given an exponentially bounded $C$-regularized semigroup $\{S(t)\}_{t \geq 0}$, one can define the Favard class of $\{S(t)\}_{t \geq 0}$, similarly as in the case of strongly continuous semigroups (see, e.g., [8], [10, Section III.3]), by

$$
\operatorname{Fav}(S(t)):=\left\{x \in X ; \sup _{t>0}\left\|\frac{1}{t}(S(t) x-x)\right\|<\infty\right\},
$$

and prove that (2.1) holds for $B \in \mathbf{L}(X)$ with

$$
\mathcal{R}(B) \subset C^{2}(\operatorname{Fav}(S(t)),
$$

and that (2.10) holds for $\mathbf{B}$ a closed linear operator in $X$ with $\mathcal{D}(\mathbf{B}) \supset \mathcal{D}(A)$ and $\mathcal{R}(\mathbf{B}) \subset C^{2}\left(\operatorname{Fav}(S(t))\right.$. When $\{S(t)\}_{t \geq 0}$ is a strong continuous semigroup, this result is essentially Theorem 2.8 .

In Example 2.9, the permissible space for $\mathcal{R}(B)$ can be so large as

$$
C\left(F_{A_{0}}\right)=\left\{f \in C^{3}(R) ; f \text { is bounded and } f^{\prime \prime \prime} \text { is Lipschitz continuous }\right\} \text {. }
$$

However, if either (2.20) or (2.14) were used, the range of $B$ would be restricted to a set which is smaller than or equal to

$$
\mathcal{R}\left(C^{2}\right)=\left\{f \in C^{4}(R) ; f \text { is bounded and } f^{(4)} \in U C_{b}(R)\right\} .
$$

It is clear that here $C\left(F_{A_{0}}\right)$ strictly contains $\mathcal{R}\left(C^{2}\right)$. This reflects the feature of Theorem 2.8, on which Example 2.9 was based.

\section{ACKNOWLEDGMENTS}

The authors are grateful to the referee for valuable suggestions.

\section{REFERENCES}

[1] Jung-Chan Chang and Sen-Yen Shaw, Perturbation theory of abstract Cauchy problems and Volterra equations, Nonlinear Analysis, TMA 30 (1997), 3521-3528. MR 98h:00034

[2] G. Da Prato, Semigruppi di crescenza n, C. R. Acad. Sci. Paris Ser. A-B 262 (1966), A996A998. MR 36:5760

[3] G. Da Prato, Semigruppi regolarizzabili, Ricerche Mat. 15 (1966), 223-248. MR 37:793

[4] E. B. Davies and M. M. Pang, The Cauchy problem and a generalization of the Hille-Yosida theorem, Proc. London Math. Soc. 55 (1987), 181-208. MR 88e:34100]

[5] R. deLaubenfels, Existence and uniqueness families for the abstract Cauchy problems, J. London Math. Soc. 44 (1991), 310-338. MR 92k:34075

[6] R. deLaubenfels, Existence families, functional calculi and evolution equations, Lecture Notes in Math., vol. 1570, Springer-Verlag, Berlin, 1994. MR 96b:47047

[7] R. deLaubenfels and F. Yao, Regularized semigroups of bounded semivariation, Semigroup Forum 53 (1996), 369-383. MR 97j:47058

[8] W. Desch and W. Schappacher, Some generation results for perturbed semigroups, Semigroup theory and applications (Proceedings Trieste 1987), P. Clément et al (Eds.), Lecture Notes in Pure and Appl. Math., vol. 116, Marcel Dekker, New York, 1989, 125-152. MR 90k:47081 
[9] O. Diekmann, M. Gyllenberg and H. R. Thieme, Perturbing semigroups by solving Stieltjes renewal equations, Diff. and Int. Eqns. 6 (1993), 155-181. MR 94b:47085

[10] K. J. Engel and R. Nagel, One-parameter semigroups for linear evolution equations, GTM, vol.194, Springer-Verlag, New York, 2000. MR 2000i:47075

[11] J. A. Goldstein, R. deLaubenfels and J. T. Sandefur, Regularized semigroups, iterated Cauchy problems and equipartition of energy, Monatsh. Math. 115 (1993), 47-66. MR 94g:47050

[12] M. Hieber, A. Holderrieth and F. Neubrander, Regularized semigroups and systems of linear partial differential equations, Ann. Scuola Norm. di Pisa 19 (1992), 363-379. MR 94a:47071

[13] M. Jung, Multiplicative perturbations in semigroup theory with the (Z)-condition, Semigroup Forum 52 (1996), 197-211. MR 96m:47073

[14] H. Kellermann and M. Hieber, Integrated semigroups, J. Funct. Anal. 84 (1989), 160-180. MR 90h:47072

[15] J. L. Lions, Les semi-groupes distributions, Portugal. Math. 19 (1960), 141-164. MR 26:611

[16] C. C. Kuo and S. Y. Shaw, C-cosine functions and the abstract Cauchy problem, I, II. J. Math. Anal. Appl. 210 (1997), 632-646, 647-666. MR 99c:47063

[17] I. Miyadera, C-semigroups and semigroups of linear operators, Differential equations (Plovdiv, 1991), World Sci. Publishing, River Edge, NJ, 1992, 133-143. MR 93k:47048

[18] H. Oka, Linear Volterra equations and integrated solution families, Semigroup Forum 53 (1996), 278-297. MR 97e:45002

[19] S. Piskarev and S. Y. Shaw, Multiplicative perturbations of $C_{0}$-semigroups and some applications to step responses and cumulative outputs, J. Funct. Anal. 128 (1995), 315-340. MR 96c: 47057

[20] S. Piskarev and S. Y. Shaw, Perturbation and comparison of cosine operator functions, Semigroup Forum 51 (1995), 225-246. MR 96h:47048

[21] N. Tanaka, C-semigroups of linear operators in Banach spaces - a generalization of the Hille-Yosida theorem, thesis, Waseda University, 1992.

[22] T. J. Xiao and J. Liang, The Cauchy problem for higher order abstract differential equations, Lecture Notes in Math. vol. 1701, Springer, Berlin, New York, 1998. MR 2001a:34099

[23] T. J. Xiao and J. Liang, Higher order abstract Cauchy problems and their existence, uniqueness families, J. London Math. Soc., to appear.

Department of Mathematics, University of Science and Technology of China, Hefei, Anhui 230026, People's Republic of China

E-mail address: xiaotj@ustc.edu.cn

Current address: Mathematisches Institut, Universität Tübingen, Auf der Morgenstelle 10, D-72076, Tübingen, Germany

E-mail address: tixi@fa.uni-tuebingen.de

Department of Mathematics, University of Science and Technology of China, Hefei, Anhui 230026, People's Republic of China

E-mail address: jliang@ustc.edu.cn

Current address: Mathematisches Institut, Universität Tübingen, Auf der Morgenstelle 10, D-72076, Tübingen, Germany

E-mail address: jili@fa.uni-tuebingen.de 